\title{
Paranoid Type Schizophrenia
}

National Cancer Institute

\section{Source}

National Cancer Institute. Paranoid Type Schizophrenia. NCI Thesaurus. Code C35006.

A subtype of schizophrenia characterized by prominent delusions (typically persecutory or grandiose) or hallucinations in the context of a relative preservation of cog nitive functioning and affect. 